\title{
An IEEE 802.16 WiMAX Module for the NS-3 Simulator
}

\author{
Jahanzeb Farooq \\ INRIA, Planete Project \\ 2004 Route Des Lucioles \\ 06902 Sophia Antipolis, France \\ jahanzeb.farooq@sophia.inria.fr
}

\author{
Thierry Turletti \\ INRIA, Planete Project \\ 2004 Route Des Lucioles \\ 06902 Sophia Antipolis, France \\ thierry.turletti@sophia.inria.fr
}

\begin{abstract}
ns-3 is a recently released next generation simulator intended as a replacement of the popular ns-2. It enables a highly rich set of features and is expected to become the first choice of the scientific community soon. ns-3 is in its evolution phase and work at different fronts is still ongoing. With the recent emergence of broadband wireless networks, simulation support for such networks, especially IEEE 802.16 WiMAX, is becoming a necessity. In this paper, we propose and discuss in detail the design and implementation of an IEEE 802.16 WiMAX module for ns-3, with the Pointto-Multipoint (PMP) mode and the WirelessMAN-OFDM PHY layer. Our module implements fundamental functions of the convergence sublayer (CS) and the MAC commonpart sublayer (CPS), including QoS scheduling services, bandwidth request/grant mechanism, and a simple uplink scheduler. The aim is to provide a standard-compliant and welldesigned implementation of the standard.
\end{abstract}

\section{Categories and Subject Descriptors}

I.6.5 [Simulation and Modeling]: Model developmentmodeling methodologies

\section{General Terms}

Algorithm, Design, Performance, Verification

\section{Keywords}

IEEE 802.16, WiMAX, network simulation, ns-3

\section{INTRODUCTION}

With the increasing demand of high data rates and larger wireless coverage, broadband wireless access networks have rapidly emerged over the last few years. The IEEE 802.16 standard [1], widely known as WiMAX (Worldwide Interoperability for Microwave Access), defines the MAC (Medium Access Control) and PHY (Physical) layers specifications for

Permission to make digital or hard copies of all or part of this work for personal or classroom use is granted without fee provided that copies are not made or distributed for profit or commercial advantage and that copies bear this notice and the full citation on the first page. To copy otherwise, to republish, to post on servers or to redistribute to lists, requires prior specific permission and/or a fee.

SIMUTools 2009, Rome, Italy

Copyright 2009 ICST 978-963-9799-45-5. the broadband wireless access networks. WiMAX offers an alternative to wired networks, such as coaxial systems using cable modems, fiber optics and DSL (Digital Subscriber Line). It offers high speed data access to large geographical areas, covering distance up to $50 \mathrm{Km}$ and supporting data rates up to $100 \mathrm{Mbps}$, depending on the distance from the base station and the underlying PHY layer. With this range and data rates WiMAX standard is capable of delivering backhaul connections for enterprise campuses, WiFi hotspots and cellular networks.

The MAC layer of WiMAX supports Point-to-Multipoint (PMP) and Mesh topologies. The wireless medium access is based on either Time Division Multiple Access (TDMA) or Frequency Division Multiple Access (FDMA), with FDD (Frequency Division Duplex) and TDD (Time Division Duplex) as duplexing techniques. Multiple PHY layer specifications are supported for LOS (Line-of-Sight) and NLOS (Non-Line-of-Sight) operational environments in $10-66 \mathrm{GHz}$ and $2-11 \mathrm{GHz}$ frequency bands, respectively. Later on the IEEE $802.16 \mathrm{e}$ amendment [3] added mobility support to WiMAX networks.

The network simulator ns-2 [6] is one of the most widely used simulators in the academic and industrial community since the last decade. The newly released ns-3 [7], primarily intended as a replacement of ns-2, is a completely new simulator rewritten from the scratch. It introduces major enhancements over its predecessor and has all the capabilities of becoming the leading network simulator in near future. A few among the many salient features of ns-3 are a completely re-written software core with fully documented API, high emphasis on conformance to real networks, easier software integration, support for simulating virtual networks, support for testbeds, attribute system for configuring simulator parameters, automatic memory management, and a configurable tracing system, etc [14]. The first release of ns3 (version ns-3.1) was made in June 2008 with support for a number of modules including CSMA, Point-to-Point, 802.11 $\mathrm{WiFi}, \mathrm{TCP}, \mathrm{UDP}$ and IPv4. The development on a number of modules is still in progress.

Currently there are a number of WiMAX modules available for ns-2, all based on PMP topology with TDD duplexing. The module proposed by Networks and Distributed Systems Laboratory (NDSL) [11], one of the first WiMAX modules available, supports, among other features, scheduling services and bandwidth management. However the module is highly simplified as it ignores several implementation details and thus is not compliant to the standard. The module proposed by National Institute of Standards and Tech- 
nology (NIST) [4] provides a number of features including OFDM PHY layer and fragmentation and de-fragmentation. However it lacks the implementation of QoS scheduling services. Later a collaborative effort between WiMAX Forum, Rensselaer Polytechnic Institute (RPI) and Washington University St. Louis (WUSTL) adds support for QoS scheduling services, ARQ mechanism and OFDMA PHY layer to this module. The updated module is now officially adopted by the WiMAX Forum [8] and is available for download for members. Note that a plug-in proposed by Telecom Bretagne [9] also extends the NIST module with QoS scheduling services feature. Finally, a recent module proposed by Computer Networks Laboratory (CNL) [10], basically based on DOCSIS module [2], supports scheduling services and bandwidth management, however it lacks implementation of a WiMAX compliant PHY layer. Unfortunately, the highly non-modular design of the NDSL and CNL modules makes them less attractive for the developers who aim to extend them with new features and plug-ins.

Our module is based on the IEEE 802.16-2004 standard and ns-3 version 3.2. The code ${ }^{1}$ is available under the GNU General Public License. It implements the PMP topology with TDD mode and aims to provide detailed and standard compliant implementation of the standard, supporting important features including QoS scheduling services, bandwidth management, uplink request/grant scheduling and the OFDM PHY layer. The module is built completely in $\mathrm{C}++$ with 36 classes and approximately 17000 lines of code. Beside the fact that it is the first WiMAX module for the ns-3 simulator, the module distinguishes itself from above modules in the respect that high attention has been put to make the design fully object-oriented, facilitating modularity, reusability, scalability and maintenance of software. Unified Modeling Language (UML) has been used for the design and analysis phase. We believe this work a significant contribution to the scientific community as it allows simulations of WiMAX networks with a more close-to-real implementation of the standard, and with the add-on flexibility of pluggingin new modules in its various components. Furthermore, with the added benefits of ns-3, the module enables features such as flexible and detailed tracing functionality, simulation of real networks and TCP/IP-stack, emulation, and defining new simulation scenarios using the Helper API.

The remainder of the paper is organized as follows. Section 2 presents a brief overview of the IEEE 802.16 standard. Section 3 presents detailed description of the proposed module including its software design, the MAC and PHY layers and the detailed operation of each component. Future development and enhancements are discussed in Section 4. Finally Section 5 concludes our work.

\section{OVERVIEW OF IEEE 802.16 STANDARD}

This section presents a brief overview of the IEEE 802.16 standard. For a more comprehensive overview please refer to [13] or [20].

The IEEE 802.16 standard defines MAC (Medium Access Control) and PHY (Physical) layers specifications for a Broadband Wireless Access (BWA) network. The physical medium is divided into frames of fixed length. Each frame is further divided into DL (downlink) and UL (uplink) sub-

${ }^{1}$ The code is available at the following URL: http://code.nsnam.org/iamine/ns-3-wimax/. frames for the downlink and uplink traffic. The structure of frame depends on the underlying PHY layer. The standard defines multiple PHY layers for different operational environments. For the $10-66 \mathrm{GHz}$ band the WirelessMANSC PHY, based on single carrier modulation, is defined. For frequencies below $11 \mathrm{GHz}$, where propagation without a direct line of sight (LOS) must be accommodated, three alternatives are provided: WirelessMAN-SCa using single carrier modulation, WirelessMAN-OFDM using Orthogonal Frequency Division Multiplexing, and WirelessMANOFDMA using Orthogonal Frequency Division Multiple Access. The standard supports two duplexing techniques: FDD, where DL and UL subframes take place at the same time but on different frequencies, and TDD, where DL and UL subframes take place at different times and usually share the same frequency.

The MAC layer is divided into three sublayers. The CS (Convergence Sublayer), MAC CPS (Common-Part Sublayer) and the Security Sublayer. The CS Sublayer is responsible of receiving PDUs (Protocol Data Units) from the higher layer, classifying these PDUs to appropriate connections, processing these PDUs and delivering them to the appropriate MAC SAP (Service Access Point). The MAC CPS (Common Part Sublayer) provides the fundamental MAC functionalities including connection establishment and management, generation of MAC management messages, SS initialization and registration, ranging, bandwidth management, service flow management and scheduling services. Two modes for sharing the wireless medium are defined: in the PMP mode Subscriber Stations (SS) communicate to each other through a Base Station (BS), and in the Mesh mode the stations are organized in an ad-hoc fashion and communicate to each other directly.

The MAC protocol is connection-oriented which means all the communication takes place in terms of connections. There are two types of connections: management connections for transmitting control messages and transport connections for data transmission. The registration phase includes an initial ranging phase in which special management messages RNG-REQ and RNG-RSP are exchanged between $\mathrm{SS}$ and $\mathrm{BS}$. The $\mathrm{BS}$ on receiving $\mathrm{RNG}-\mathrm{REQ}$ examines parameters such as power level and if acceptable allocates a set of management connections to the SS. These connections are then used to transmit and receive control messages.

The MAC defines a set of control messages, called MAC management messages. The most important of these messages are DL-MAP, UL-MAP, DCD and UCD. At the beginning of each frame the BS broadcasts the DL-MAP and UL-MAP messages that define access to the DL and UL subframes of the current frame. These messages contain a set of information elements (IE), each notifying an SS of the DL or UL grant allocated to it. These are followed by DCD and UCD messages which contain general information about the channel and a set of burst profiles, each burst profile defining a set of parameters that must be used to send or receive data in a given DL or UL grant.

All the data traffic is carried on transport connections. There is a one-to-one relationship between a transport connection and a service flow. A service flow defines a unidirectional flow of data traffic and binds it with a set of QoS parameters. A wide range of QoS parameters are supported by the standard. Special MAC management messages DSAREQ and DSA-RSP are exchanged between SS and BS to 
create a service flow.

The standard defines four scheduling services (or QoS classes), named UGS (Unsolicited Grant Service), rtPS (RealTime Polling Service), nrtPS (Non-Real-Time Polling Service) and $\mathrm{BE}$ (Best Effort). UGS is designed for real-time applications with fixed size packets. UGS flows are allocated unsolicited grants on periodic basis. rtPS is designed for real-time applications that generate variable size packets on periodic basis. Unlike UGS, a rtPS flow is polled by the BS on periodic basis allowing it to send bandwidth requests. The request is then serviced based on the available bandwidth. Compared to rtPS, nrtPS is designed for more delay-tolerant applications. A nrtPS flow is ensured a minimum bandwidth by polling it on regular basis even in congestions conditions. Finally, BE (Best Effort) is designed for best effort traffic such as HTTP and Email and does not guarantee a minimum bandwidth. The $802.16 \mathrm{e}$ amendment introduces an additional class named ertPS (Extended RealTime Polling Service) that combines features of UGS and rtPS by allowing unsolicited grants for variable packet size applications.

Bandwidth is requested by sending a bandwidth request packet. It is sent either in a special transmission opportunity allocated in UL subframe or in an uplink grant allocated to the SS. BS then responds with a grant allocated in subsequent frames. The uplink scheduler at the BS decides which SSs to allocate bandwidth in the next frame based on requests. Note that bandwidth is requested on per connection basis but allocated on per SS basis. This is then the responsibility of the SS scheduler to decide which connection will transmit in a given grant. Similarly the BS scheduler selects packets that will be transmitted in DL subframe. Note that standard does not define any scheduling algorithm for these schedulers and leaves this up to the manufacturers to decide.

\section{THE PROPOSED IEEE 802.16 MODULE}

The proposed module is mainly composed of three layers: the CS (Convergence Sublayer), the MAC CPS (CommonPart Sublayer) and the PHY layer. At the PHY layer two different PHY implementations are supported. Figure 1 presents a block diagram of the module, illustrating some of its key components and their interaction. Subsequent sections provide detailed description of the three layers and the key components.

\subsection{Software Design}

The design of the module thoroughly follows object-oriented analysis and design methodology in order to benefit from the classic characteristics of object-oriented software construction including modularity, polymorphism, encapsulation and inheritance, and to conform to fully object-oriented design of ns-3. UML (Unified Modeling Language) has been adopted especially for the Design and Analysis phases of the SDLC (System Development Life Cycle). More specifically, software construction techniques presented in [18] have been widely followed. The class diagram of the module is presented in Figure 2. Note that the diagram only shows important data members and functions and details such as multiplicity have been omitted due to limited space. As seen in the figure, the module is composed of several $\mathrm{C}++$ classes each representing a core component of the system and taking care of a particular functionality. Responsibilities have been carefully delegated to different classes/components to ensure high cohesion and low coupling. High attention has been put to design an API that ensures reusability, scalability as well as maintainability of the software. The modular design facilitates extension of the software in future. Additions of new components and plug-ins, such as new PHY or scheduler implementations, can easily be made.

\subsection{The Convergence Sublayer}

The 802.16 MAC layer is divided in to two sublayers. The Convergence Sublayer and the core MAC layer called MAC Common-Part Sublayer. CS is further sub-divided into two types: the Packet CS and the ATM CS. Our module implements the Packet CS, designed to work with the packet-based protocols at higher layers. The CS is responsible of receiving packet from the higher layer and from peer stations, classifying packets to appropriate connections (or service flows) and processing packets. It keeps a mapping of transport connections to service flows. This enables the MAC CPS identifying the QoS parameters associated to a transport connection and ensuring the QoS requirements. The CS currently employs a simple classifier based on destination MAC address. Support for other standard compliant classifiers is aimed in future (see Section 4). Note that the optional feature of PHS (Payload Header Suppression), which allows suppressing the repetitive portion of the payload headers, is not implemented by the module.

\subsection{The MAC Sublayer}

The MAC CPS is the main sublayer of the IEEE 802.16 $\mathrm{MAC}$ and performs the fundamental functions of the MAC. The module implements the PMP mode. In PMP mode BS is responsible of managing communication among multiple SSs. The key functionalities of the MAC CPS include framing and addressing, generation of MAC management messages, SS initialization and registration, service flow management, bandwidth management and scheduling services.

Class WimaxNetDevice in Figure 2 represents the MAC layer of a WiMAX network device. This class extends the NetDevice class of the ns-3 API that provides abstraction of a network device. WimaxNetDevice is further extended by BaseStationNetDevice and SubscriberStationNetDevice classes, defining MAC layers of BS and SS, respectively. Besides these main classes, the key functions of MAC are distributed to several other classes to achieve software characteristics discussed in Section 3.1. As seen from Figure 2, some of the key classes that implement the MAC functions include Link Manager (for both SS and BS), Uplink Scheduler, Scheduler (for both SS and BS), Connection Manager, Service Flow Manager, Burst Profile Manager and Bandwidth Manager.

\subsubsection{Framing and Management Messages}

The MAC is partitioned into frames of fixed duration. The module implements a frame as a fixed duration of time, i.e., frame boundaries are defined with respect to time. Each frame is further subdivided into DL and UL subframes. The module implements the TDD mode where DL and UL operate on same frequency but are separated in time. A number of DL and UL bursts are then allocated in DL and UL subframes, respectively. Since the standard allows sending and receiving bursts of packets in a given DL or UL burst, the unit of transmission at the MAC layer is a packet burst. The module implements a special Packet Burst data structure for this purpose (see Figure 2). A packet burst is essen- 


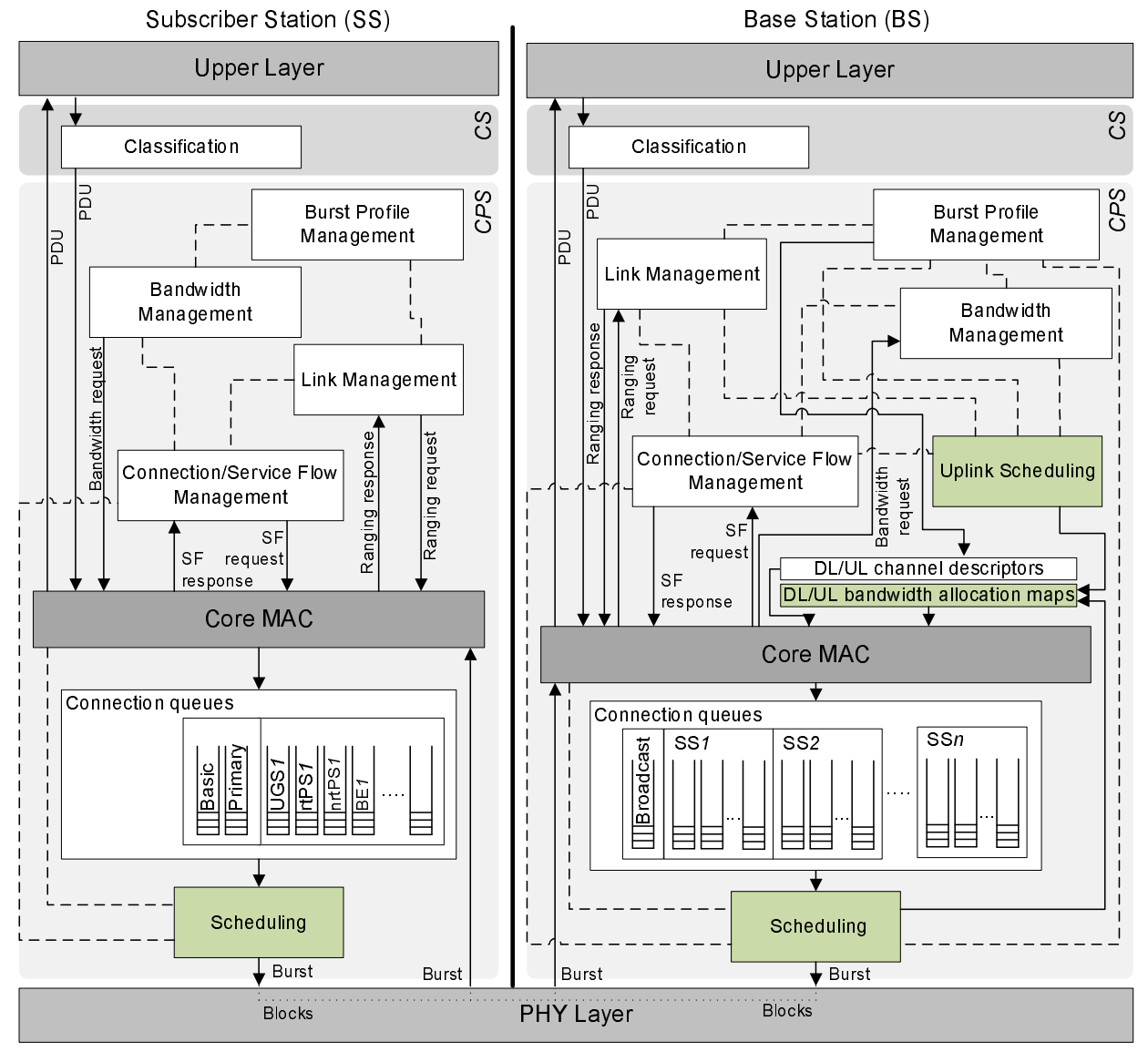

Figure 1: Block diagram of the proposed WiMAX module.

tially a list of packets. As seen in Figure 1, the BS downlink and uplink schedulers (represented by classes BS Scheduler and Uplink Scheduler in Figure 2) are responsible of generating DL and UL subframes, respectively. In the case of DL, the subframe is simulated by transmitting consecutive bursts (instances of PacketBurst class). In case of UL, the subframe is divided, with respect to time, into a number of slots. The bursts transmitted by the SSs in these slots are then aligned to slot boundaries. The frame is divided into integer number of symbols and PS (Physical Slots) which helps in managing bandwidth more effectively. The number of symbols per frame depends on the underlying implementation of the PHY layer (see Section 3.4). The size of a DL or UL burst is specified in units of symbols. Figure 3 shows the format of implemented MAC frame and the key management messages DL-MAP, UL-MAP, DCD and UCD.

MAC management messages play fundamental role in IEEE 802.16 MAC. A number of management messages are defined. These messages only carry control information and are transmitted on management connections (including predefined broadcast and multicast connections). The most important of these messages are DL-MAP, UL-MAP and DCD and UCD. DL-MAP and UL-MAP are called DL and UL bandwidth allocation maps and define access to the downlink and uplink, respectively. DCD and UCD are called channel descriptors and contain information about the chan- nel and how data shall be transmitted/received on it. Next subsection describes these messages in further detail. In addition to these messages, the standard defines separate sets of management messages to carry specific tasks such as ranging, registration, service flow management, burst profile management and so on. The module currently implements the following management messages: DL-MAP, UL-MAP, DCD, UCD, RNG-REQ, RNG-RSP, DSA-REQ, DSA-RSP and DSA-ACK. The module implements these messages, as well as the regular packets and the 802.16 MAC headers, Generic MAC Header, Bandwidth Request Header, and Grant Management Subheader, utilizing ns-3's Packet/Header API, that, among other features, allows bit-by-bit serialization and de-serialization of the packet bytes with the help of the underlying byte buffer. This serialized representation of the data in the packet is done to match real network packets, in order to facilitate features such as emulation.

\section{DL-MAP/UL-MAP and DCD/UCD Messages}

Figure 3 shows the format of the DL-MAP, UL-MAP, DCD and UCD messages. DL-MAP and UL-MAP messages define access to the DL and UL subframes of the MAC frame. The Allocation Start Time field of UL-MAP indicates the starting time of the UL subframe, relative to the beginning of the DL subframe. As seen in figure, DL-MAP and UL-MAP contain a set of special data structures, called IE (Informa- 


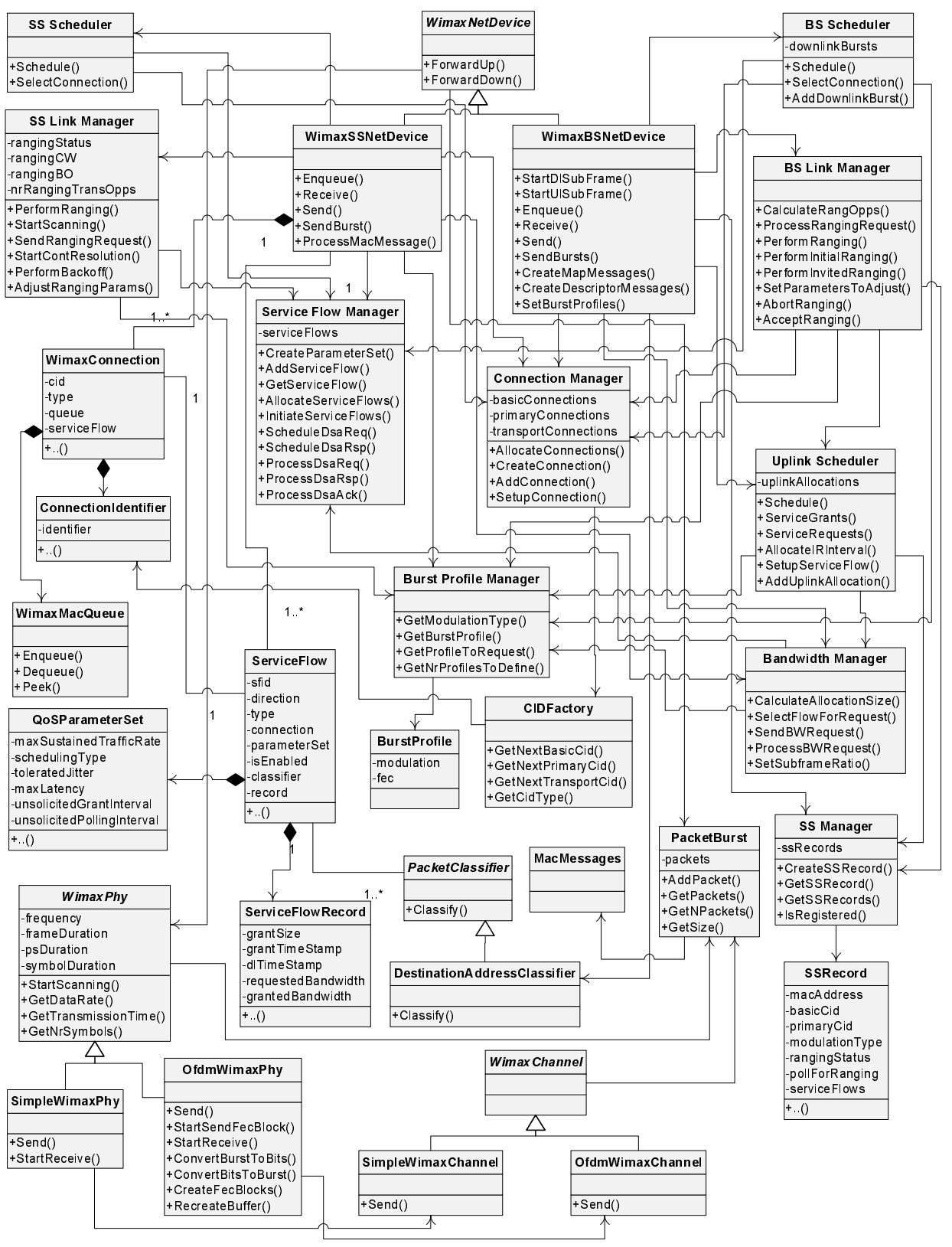

Figure 2: Class diagram of the proposed WiMAX module.

tion Element). Each IE notifies an SS (or set of SSs) of an upcoming DL or UL burst (or grant).

The detailed contents of DL-MAP and UL-MAP IE are as follows. Each DL-MAP IE includes, among others, a $C I D$ field identifying an SS (or a set of SSs in case of multicast or broadcast) that will be receiving the corresponding DL burst, and a DIUC (Downlink Interval Usage Code) indicating a burst profile to be used to receive the burst. A burst profile defines a set of PHY parameters that the SS must use to send or receive packets. DL and UL burst profiles are defined in DCD and UCD, respectively. DL-MAP IE also includes a Start Time field that allows SSs to go into sleep mode and wake up when start time is elapsed. This field is currently not utilized as the module does not implement the power saving feature. Similarly, each UL-MAP IE notifies of an upcoming UL grant. Besides the CID of the SS that is allocated the grant, it includes a Start Time field that marks the beginning of the grant, a duration field indicating duration of the grant, and a UIUC (Uplink Interval Usage Code) that maps to a UL burst profile in the UCD message.

DCD and UCD messages include information about the channel as well as a set of burst profiles. The Burst Profile Manager component (see Figures 1 and 2) is responsible of defining burst profiles and creating these messages. 


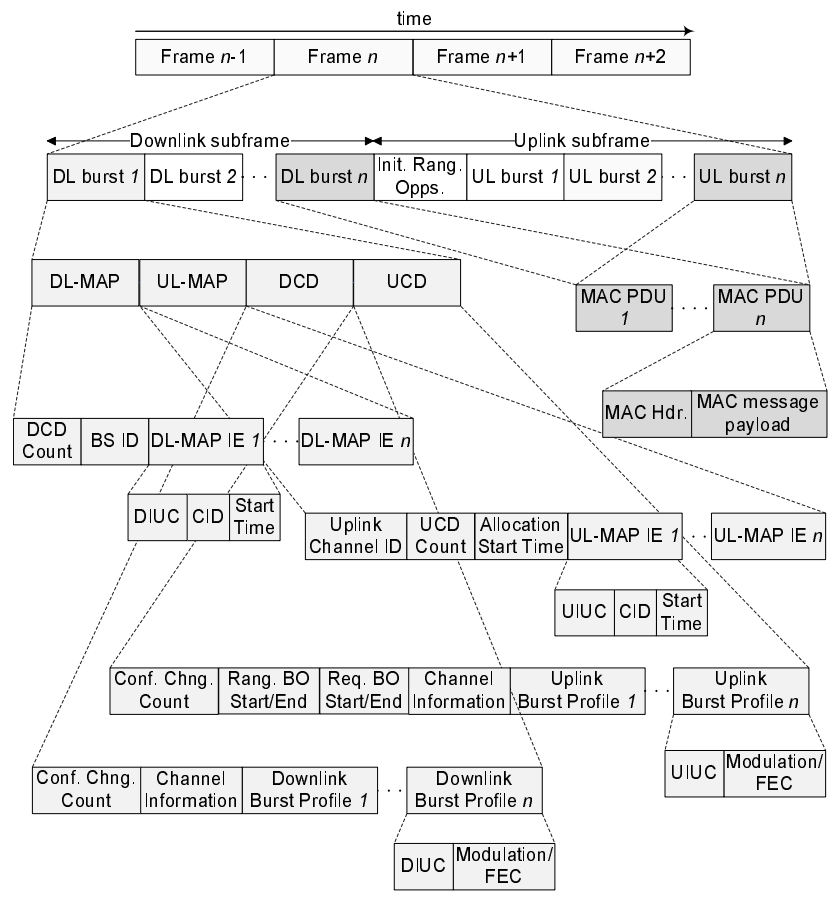

Figure 3: Format of the MAC frame and key managements messages.

UCD message contains important parameters including the backoff limits for the contention ranging and bandwidth request processes, and size of the ranging and the bandwidth request transmission opportunities. DCD message contains information including the size of the TTG (Transmit/receive Transition Gap) and RTG (Receive/transmit Transition Gap), a number identifying the current frame, and the duration of the frame. The burst profile data structure contains a combination of modulation and FEC code and a DIUC/UIUC uniquely identifying the burst profile. For the WirelessMAN-OFDM PHY layer, seven combinations of modulation and FEC codes are supported (see Table 4).

The four management messages described above are always sent in the first burst in the DL subframe, that contains broadcast messages. In current implementation, DL-MAP and UL-MAP are sent in every frame and DCD and UCD are sent on random basis simulating the real network where definitions of burst profiles may update with time. The rest of the bursts in the subframe are regular data bursts directed towards individual SSs (or a set of SSs).

As an SS receives a DL-MAP message, it iterates through the DL-MAP IEs to determine if a DL burst is directed towards it following the current broadcast burst. If so, it receives the burst by decoding it using the parameters in the corresponding burst profile. Similarly, as an SS receives a UL-MAP indicating an UL grant for it, the SS starts sending its pending data as the start time of the grant elapses.

\subsubsection{Outbound and Uplink Schedulers}

The standard defines two types of schedulers. The outbound scheduler that selects packets from the outbound queues to send, and the BS uplink scheduler that allocates bandwidth to SSs in the UL, to allow them sending their packets. Classes SSScheduler and BSScheduler in Figure 2 implement the outbound schedulers of SS and BS, and class UplinkScheduler implements the BS uplink scheduler. The BS Scheduler (also referred to as Downlink Scheduler) schedules, out of the various connections queues, packets to send in the DL subframe. The SS Scheduler schedules, out of its connections queues, packets to send in an UL grant allocated to it. The Uplink Scheduler manages UL bandwidth, with the help of Bandwidth Manager, and schedules SSs that will be allocated UL grants based on the QoS requirements of the their service flow(s) and bandwidth requests (see Sections 3.3.5 and 3.3.7). Note that the standard does not define any particular scheduling algorithm for these schedulers and leaves it to the manufacturers to decide (see Section 3.3.6).

The Downlink Scheduler and Uplink Scheduler components of BS are invoked at the beginning of every MAC frame. These two schedulers are responsible of creating the DL and UL subframes (or the DL-MAP and UL-MAP messages), respectively. The Downlink Scheduler creates the bursts to send in the DL subframe by selecting packets, from the queues of all SSs registered with the BS. A burst may contain multiple packets for the same SS the burst is directed towards. As seen in Figure 1, the BS performs scheduling on queue per connection per SS basis (see Section 3.3.4 for details on connections) instead of maintaining a single queue per SS or per scheduling service type. This design has been preferred over the latter due to expensive computations it involves. After creating the bursts, it creates a list of DLMAP IEs, each IE pointing to a burst. These pre-created DL-MAP IEs are then used to create the DL-MAP in the first burst of DL subframe. The pre-created bursts are sent following the first burst. Similarly, the Uplink Scheduler creates a list of UL-MAP IEs and based on that the UL-MAP is created. The SS Scheduler is invoked every time a UL grant is allocated to the SS.

\subsubsection{Network Entry and Initialization}

The network entry and initialization phase is basically divided into two sub-phases, (1) scanning and synchronization and (2) initial ranging. The entire phase is performed by the Link Manager component of SS and BS.

\section{Scanning and Synchronization}

Once an SS wants to join the network, it first scans the downlink frequencies to search for a suitable channel. The search is complete as soon as it detects a PHY frame. The next step is to establish synchronization with the BS. Once SS receives a DL-MAP message the synchronization phase is complete and it remains synchronized as long as it keeps receiving DL-MAP and DCD messages. After the synchronization is established, SS waits for a UCD message to acquire uplink channel parameters. Once acquired, the first sub-phase of the network entry and initialization is complete.

\section{Initial Ranging and Registration}

Once synchronization is achieved, the SS waits for a ULMAP message to locate a special grant, called initial ranging interval, in the UL subframe. This grant is allocated by the BS Uplink Scheduler at regular intervals, and is always the first grant if present. It is always directed towards the Broadcast CID and uses the predefined most robust burst 
profile with BPSK $1 / 2$ modulation/FEC. Currently this interval is set to $0.5 \mathrm{~ms}$, however the user is enabled to modify its value from the simulation script.

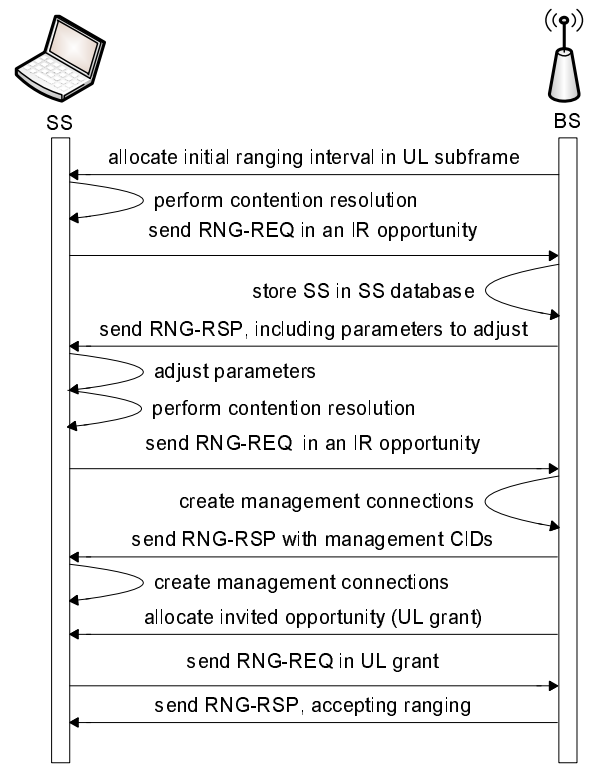

Figure 4: Initial ranging process.

Initial ranging interval is composed of one or more transmission opportunities. The size of a opportunity is fixed, it is determined by the BS and allows sufficient time to send a RNG-REQ message including the overhead (SS learns its size from UCD message). Once located, the SS sends a RNG-REQ message after performing a backoff based contention resolution process.

The BS upon receiving a RNG-REQ responds with a RNGRSP message indicating if the ranging is accepted or not, or indicating the SS to adjust its timing offset/power parameters. At the same time the SS Manager component adds the SS in its database of SSs. The SS Manager component is extensively used by the BS to manage SSs in various of its operations. The exchange of RNG-REQ and RNG-RSP messages continues until the ranging is accepted or rejected. Figure 4 illustrates a simplified scenario of initial ranging phase. Note that initial ranging is initially performed on the predefined initial ranging connection until BS allocates the Basic and Primary management connections to the SS through the RNG-RSP message. From this point onwards $\mathrm{SS}$ is allocated an invited (or unicast) ranging opportunity (basically a UL grant), to continue ranging process.

\subsubsection{Connections and Addressing}

All communication at the MAC layer is carried in terms of connections. The standard defines a connection as a unidirectional mapping between the SS and BS's MAC entities for the transmission of traffic. As discussed in Section 2, the standard defines two types of connections: management connections for transmitting control messages and transport connections for data transmission. A connection is identified by a 16-bit CID (Connection Identifier). Classes WimaxConnection and ConnectionIdentifier in Figure 2 implement the connection and CID, respectively. Note that each con- nection maintains its own transmission queue where packets to transmit on that connection are queued. The Connection Manager component of BS is responsible of creating and managing connections for all SSs.

The two key management connections defined by the standard, namely the Basic and Primary management connections, are created and allocated to the SS during the ranging process. Basic connection plays an important role throughout the operation of SS also because all (unicast) DL and UL grants are directed towards SS's Basic CID. In addition to management connections, an SS may have one or more transport connections to send data packets (see Section 3.3.5).

The Connection Manager component of SS manages the connections associated to SS. As defined by the standard, a management connection is bidirectional, i.e., a pair of downlink and uplink connections is represented by the same CID. This feature is implemented in a way that one connection (in DL direction) is created by the BS and upon receiving the CID the SS then creates an identical connection (in UL direction) with the same CID.

Table 1: CID value ranges.

\begin{tabular}{|l|l|}
\hline CID & Value \\
\hline Initial Ranging & 0x000 \\
\hline Basic & 0x000 $-m$ \\
\hline Primary & $m+1-2 m$ \\
\hline Transport and Secondary & $2 m+1-$ 0xFE9F \\
\hline Multicast & 0xFEA0 - 0xFEFE \\
\hline Multicast Polling & 0xFF00 - 0xFFF9 \\
\hline Broadcast & 0xFFFF \\
\hline
\end{tabular}

Note that since MAC is completely connection oriented, the MAC address of SS is only used during the initial ranging phase before management CIDs are assigned. Once assigned, management and transport CIDs are used to address the SS and individual transport connections associated to SS. The CID is included in the MAC header of each packet to indicate the connection the packet would be sent on. The CID Factory component (class CidFactory in Figure 2) at BS generates unique CIDs for all types of connections. Table 1 lists ranges of CID values for each type of connection as defined by the standard.

\subsubsection{Service Flow Creation}

The standard defines a service flow as an unidirectional flow of MAC PDUs on a transport connection that ensures specific QoS requirements. A service flow is identified by a 32-bit Service Flow ID (SFID) and is associated to exactly one transport connection and QoS parameter set. Classes ServiceFlow and QoSParameterSet in Figure 2 implement service flow and QoS parameter set, respectively. The Service Flow Manager component at the BS is responsible of creating and managing service flows.

The standard defines two mechanisms of service flow creation: a SS-initiated mechanism where SS requests the BS to create a service flow, and a BS-initiated mechanism where BS automatically creates service flow for the SS. The module implements the first of the above mechanisms. In this mechanism the user is allowed to setup, for each application he/she intends to simulate, a QoS parameter set that matches the QoS requirements of the application. Once the 
$\mathrm{SS}$ is registered, it sends a request (a DSA-REQ message) to the BS, along with the predefined QoS parameter set, to create the service flow. The BS examines the parameters and assuming that all parameters are supported, creates a service flow (and transport connection) and responds with a DSA-RSP message. The SS may now start using the service flow to send/receive data.

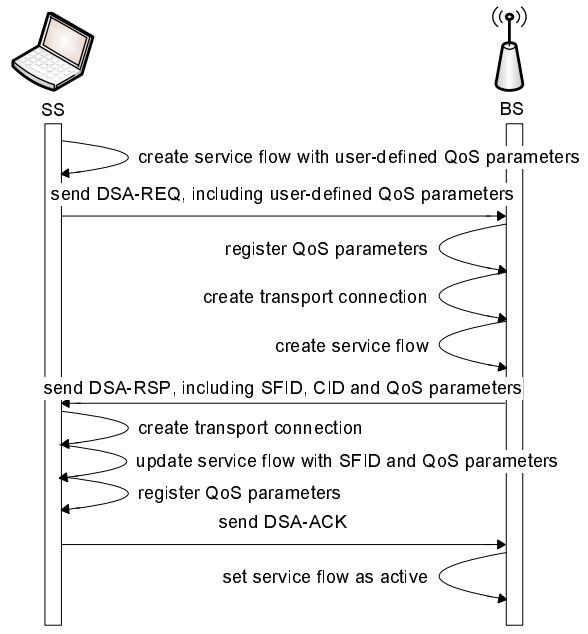

Figure 5: Service flow creation process.

Figure 5 shows the main steps of service flow creation process. All DSA messages are sent on the Primary management connection. The entire service flow creation process is performed by the Service Flow Manager components of SS and BS. Multiple service flows per SS are allowed and the above message exchange is invoked for each service flow. Note that a pair of service flows must be created with the same QoS parameters for both sender and receiver. Also note that an SS can not start data transmission unless it has acquired at least one service flow.

\subsubsection{Scheduling Services}

The module supports the four scheduling services (UGS, rtPS, nrtPS, BE) defined by the 802.16-2004 standard. These scheduling services behave differently with respect to how they request bandwidth as well as how the it is granted. Each service flow is associated to exactly one scheduling service, and the QoS parameter set associated to a service flow actually defines the scheduling service it belongs to. Table 2 lists the mandatory QoS parameters defined by the standard that must be supported by a scheduling service. When a service flow is created the Uplink Scheduler calculates necessary parameters such as grant size and grant interval based on QoS parameters associated to it. For the real-time services (UGS and rtPS) the BS then allocates grants/polls on regular basis, based on the calculated interval. For the nonreal-time services (nrtPS and $\mathrm{BE}$ ) only minimum reserved bandwidth is guaranteed, if available after servicing realtime flows. Note that not all of the listed parameters are currently utilized by the scheduler. Also note that currently only service flows with fixed packet size are supported.

As discussed in Section 3.3.2, the standard does not suggest any scheduling algorithm for the schedulers. The implemented schedulers work based on a simple priority-based
Table 2: Scheduling services and suggested parameters.

\begin{tabular}{|l|l|}
\hline Scheduling Service & Parameter \\
\hline \multirow{4}{*}{ UGS } & SDU Size \\
\cline { 2 - 2 } & Minimum Reserved Traffic Rate \\
\cline { 2 - 2 } & Maximum Latency \\
\cline { 2 - 2 } & Request/Transmission Policy \\
\cline { 2 - 2 } & Unsolicited Grant Interval \\
\hline \multirow{5}{*}{ rtPS } & Maximum Latency \\
\cline { 2 - 2 } & Minimum Reserved Traffic Rate \\
\cline { 2 - 2 } & Maximum Sustained Traffic Rate \\
\cline { 2 - 2 } & Traffic Priority \\
\cline { 2 - 2 } & Request/Transmission Policy \\
\cline { 2 - 2 } & Unsolicited Polling Interval \\
\hline \multirow{4}{*}{ nrtPS } & Minimum Reserved Traffic Rate \\
\cline { 2 - 2 } & Maximum Sustained Traffic Rate \\
\cline { 2 - 2 } & Traffic Priority \\
\cline { 2 - 2 } & Request/Transmission Policy \\
\hline \multirow{4}{*}{ BE } & Maximum Sustained Traffic Rate \\
\cline { 2 - 2 } & Request/Transmission Policy \\
\hline
\end{tabular}

FCFS (First Come First Served) algorithm. For the uplink scheduling, priority is given starting from highest priority scheduling type to the lowest priority one (UGS > rtPS > nrtPS $>$ BE). Among the flows of same scheduling type, priority is given on FCFS basis. For the BS and SS (outbound) schedulers, higher priority is given to the initial ranging and management connections, i.e., broadcast $>$ initial ranging $>$ basic $>$ primary $>$ UGS $>$ rtPS $>$ nrtPS $>$ BE. Following is a brief overview of the implemented scheduling.

\section{UGS Scheduling}

UGS (Unsolicited Grant Service) is designed for real-time applications with fixed packet size. It guarantees bandwidth on real-time basis, by allocating the flow UL grants on regular basis. The key parameters for this service are tolerated jitter, maximum latency and minimum reserved traffic rate. The tolerated jitter parameter specifies the maximum tolerated delay variation and maximum latency specifies the maximum delay between arrival and forwarding of the packet (at the BS side). The Minimum reserved traffic rate parameter specifies the minimum bandwidth (in bps) that must be reserved for the flow. These parameters are supplied by the user while setting up the service flow. Based on these parameters, the BS calculates the interval, called unsolicited grant interval, when the service flow must be allocated a grant. The Downlink Scheduler, while scheduling UGS traffic, must ensure that the latency requirements of the flow are met. Every time the scheduler is invoked, the queues are refreshed and packets whose latency deadlines have elapsed are dropped.

\section{rtPS Scheduling}

rtPS (Real-Time Polling Service) is designed for real-time applications with variable packet size, and is ensured UL grants on periodic basis. For a rtPS flow, the BS polls the SS with a special grant, referred to as request opportunity, allowing it to send bandwidth request. The BS in response allocates sufficient bandwidth (see Section 3.3.7 for details) in the subsequent frames. This mechanism enables the SS to specify the size of the grant, to meet flow's variable size 
traffic needs. However, compared to UGS, rtPS incurs more overhead due to the bandwidth request packets. The key parameters for rtPS service are maximum latency and minimum reserved traffic rate. The BS, based on these parameters, calculates the polling interval (unsolicited polling interval). The Uplink Scheduler ensures the flow of the minimum bandwidth. Like UGS, the Downlink Scheduler makes sure that the latency requirements of the flow are met.

\section{nrtPS Scheduling}

The only difference between rtPS and nrtPS (Non-RealTime Polling Service) is that the latter is designed for nonreal-time applications. nrtPS flows are not polled on periodic basis and instead only when sufficient bandwidth is available. SS then specifies the size of the grant through the bandwidth request packet and BS responds by allocating sufficient bandwidth only when available. However the BS ensures the flow of the minimum bandwidth (based on minimum reserved traffic rate parameter) by periodically monitoring total bandwidth granted to the flow. Currently this period is set to one second. nrtPS does not use the maximum latency parameter.

\section{BE Scheduling}

$\mathrm{BE}$ (Best Effort) service, as the name suggests, is designed for the low priority best effort applications. For a BE flow, the scheduler allocates bandwidth only when sufficient bandwidth is available after servicing higher priority flows. No minimum bandwidth is guaranteed for this service.

\subsubsection{Bandwidth Request and Grant Mechanism}

Bandwidth request and grant mechanism is a key feature of the WiMAX MAC since all three non-UGS scheduling types explicitly request for bandwidth by sending a bandwidth request packet. The bandwidth request and grant mechanism is implemented through the Bandwidth Manager components of BS and SS.

The mechanism works as follows. An SS with rtPS, nrtPS or $\mathrm{BE}$ flow is polled by the $\mathrm{BS}$, by allocating it a request opportunity, on periodic basis. A request opportunity is basically a UL grant, directed towards SS's Basic CID, however it cannot be used to send data packets. It is distinguished (from regular UL grants for data) by the predefined most robust burst profile that must be used to send bandwidth request in it. The size of this grant is fixed, allowing sufficient bandwidth to send a bandwidth request packet. A bandwidth request packet includes a special Bandwidth Request Header. The BR field of this header is used to notify the $\mathrm{BS}$ the requested bandwidth (in units of bytes). As the SS receives a poll, the $S S$ Scheduler iterates through SS's nonUGS flows and for a flow with pending packets, it sets the $\mathrm{BR}$ field to the size of the packet in front of the queue. CID field of the header is set to flow's CID. The bandwidth request packet is then sent and the BS responds by allocating sufficient bandwidth in subsequent frames. Figure 6 illustrates the main steps of bandwidth request/grant process. The inclusion of Bandwidth Request Header is mutually exclusive with Generic MAC Header. The standard defines an optional piggyback request feature that allows sending bandwidth request piggybacked with data. However this feature is not supported in our implementation.

As discussed above, bandwidth is always requested on a per flow basis but granted on per SS basis. The SS Sched-

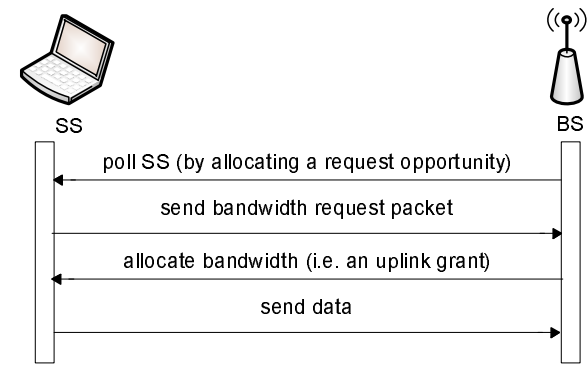

Figure 6: Bandwidth request/grant process.

uler then decides which flow shall send its data in the given grant. For the UGS and rtPS flows, the scheduler utilizes the unsolicited grant interval and unsolicited polling interval parameters, respectively, by selecting the flow whose interval is closest to elapse. The non-real-time services (nrtPS and $\mathrm{BE}$ ) are serviced only if sufficient bandwidth is left.

Since all SSs with at least one UGS service flow are automatically allocated grants to service their UGS traffic, if such an SS has a non-UGS flow, it must notify this to the $\mathrm{BS}$ in order to be polled to send bandwidth requests. This is done by setting the poll-me field of a special type of subheader, called Grant Management Subheader. This subheader is included together with the Generic MAC Header. The BS upon receiving a UGS packet with the poll-me field set, starts polling the SS for non-UGS flows.

\subsubsection{MAC Queue}

As discussed in Section 3.3.4, each connection maintains its own transmission queue. In order to support a number of MAC specific features, the module implements its own specialized queue (class WimaxMacQueue in Figure 2). This queue data structure supports storing packets and headers separately, enabling the schedulers to determine in advance the CID of the connection a packet will be sent on, without dequeueing the packet, by peeking the header. Header is appended to packet only when packet is dequeued and is about to be sent, following the scheduling phase. Furthermore, in order to implement the bandwidth request/grant mechanism, the queue data structure is designed to support both types of packets, the regular packets and the bandwidth request packets. As discussed in Section 3.3.7, the two packet types contain two different types of headers. Note that the queue still functions in FIFO (First In First Out) fashion for both types of packets, in that the queue is iterated and the first packet of the respective type is accessed/dequeued. Also note that this functionality is only used by the SS, as the BS does not send bandwidth request packets.

\subsection{The PHY Layer}

The module provides two different versions of the PHY layer. The first is a basic PHY implementation which simply forwards bursts received by the MAC layer ignoring any underlying PHY layer details. Class SimpleWimaxPhy in Figure 2 represents this simple PHY layer. The second is the OFDM PHY layer based on the WirelessMAN-OFDM specification, represented by class OfdmWimaxPhy in the figure. Since the OFDM PHY deals with the channel encoding blocks (or FEC blocks), packet bursts are converted to bit-streams and then splitted into smaller FEC blocks. 
The PHY currently works at $5 \mathrm{GHz}$ license-free band assuming $20 \mathrm{MHz}$ channel bandwidth and a 10 milliseconds frame following the parameters used in [15]. This results in OFDM symbol duration of $13.89 \mu$ s, hence 720 symbols per frame. Table 3 lists the key PHY parameters.

Table 3: OFDM PHY parameters.

\begin{tabular}{|l|l|l|}
\hline Parameter & Calculation & Value \\
\hline Frequency band & & $5 \mathrm{GHz}$ \\
\hline Frame duration & & $10 \mathrm{~ms}$ \\
\hline Bandwidth $B W$ & & $20 \mathrm{MHz}$ \\
\hline Carriers $N_{F F T}$ & & 256 \\
\hline Data carriers & & 192 \\
\hline Sampling factor $n$ & & $144 / 125$ \\
\hline Sampling frequency $F_{s}$ & $n . B W$ & $23.04 \mathrm{MHz}$ \\
\hline Subcarrier spacing $f \Delta$ & $F_{s} / N_{F F T}$ & 90000 \\
\hline$G$ & $T_{g} / T_{b}$ & $1 / 4$ \\
\hline Useful symbol time $T_{b}$ & $1 / f \Delta$ & $11.11 \mu \mathrm{s}$ \\
\hline Cyclic Prefix time $T_{g}$ & $G . T_{b}$ & $2.78 \mu \mathrm{s}$ \\
\hline OFDM symbol time $T_{s}$ & $T_{b}+T_{g}$ & $13.89 \mu \mathrm{s}$ \\
\hline PS duration & $4 / F_{s}$ & $0.1736 \mu \mathrm{s}$ \\
\hline
\end{tabular}

The PHY utilizes an external OFDM PHY module [12], which implements the fundamental phases of the OFDM PHY layer following the specification. This module utilizes external mathematics and signal processing library IT ++ [5]. The FEC blocks are forwarded to this module which then performs the fundamental operations of channel encoding, randomization, interleaving and modulation etc, and transmits it to the channel (and the reverse process at the receiving side). In this respect the OfdmWimaxPhy class also serves as an interface to this external OFDM module.

WirelessMAN-OFDM PHY supports seven modulations together with FEC coding rates. Table 4 lists these modulations with the corresponding data rates.

Table 4: Modulation and coding rates and corresponding data rates.

\begin{tabular}{|l|l|l|l|}
\hline Modulation & $\begin{array}{l}\text { Coding } \\
\text { Rate }\end{array}$ & $\begin{array}{l}\text { Block } \\
\text { Size }\end{array}$ & $\begin{array}{l}\text { Data Rate } \\
\text { [Mbps }]\end{array}$ \\
\hline BPSK & $1 / 2$ & 12 & 6.91 \\
\hline QPSK & $1 / 2$ & 24 & 13.82 \\
\hline QPSK & $3 / 4$ & 36 & 20.74 \\
\hline 16 QAM & $1 / 2$ & 48 & 27.65 \\
\hline 16 QAM & $3 / 4$ & 72 & 41.47 \\
\hline 64 QAM & $2 / 3$ & 96 & 55.30 \\
\hline 64 QAM & $3 / 4$ & 108 & 62.21 \\
\hline
\end{tabular}

\subsubsection{Operation}

The OFDM PHY layer works as follows. Once it receives a packet burst from the MAC layer, this burst is converted into a plain stream of bits. This bit-stream is splitted into smaller FEC blocks depending on the modulation scheme currently being used. Table 4 lists block sizes for the seven modulations as defined by the standard. These blocks are then forwarded to the OFDM module one by one. The OFDM module performs the fundamental PHY operations on the block and forwards it to the channel layer which transmits the block. The PHY layer at the receiving side then performs the reverse operations of concatenating the blocks to bit-stream and converting it back to packet burst. The burst is then forwarded to the MAC layer.

\subsubsection{Design}

The design of the PHY layer facilitates addition of new PHY layers in future. As seen in Figure 2, WimaxPhy is defined as an abstract class and must be extended to add a new PHY implementation, as currently done for simple PHY and OFDM PHY implementations. Similarly, WimaxChannel provides abstract implementation of the channel, to allow addition of PHY-compatible channel. Currently the simple PHY implementation uses the compatible Simple WimaxChannel, which transmits packet bursts, and the OFDM PHY uses Ofdm WimaxChannel, which transmits FEC blocks. This design enables users to easily plug-in or switch to different PHY layers from the simulation script.

\section{LIMITATIONS AND FUTURE WORK}

The module currently lacks a full implementation of the classifier as well as support for fragmentation and defragmentation of PDUs. Due to the lack of classifier, the module currently allows simulating only one service flow per SS. A classifier is used to map incoming packets to appropriate connections (or service flows) based on a set of criteria. An incoming packet from higher layer does not contain sufficient information to map it to a service flow of a particular scheduling service. To make it work some updates needs to be done in conjunction with the higher layers, particularly the Application layer. It should be noted that due to these complexities, none of the available WiMAX modules discussed in Section 1 implements a full, standard-complaint classifier.

IP over IEEE 802.16 [16] is a much highlighted area of discussion ever since the release of the standard. One of the key issues when deploying IP over IEEE 802.16 networks is how to configure an IP Subnet over a MAC which is essentially connection-oriented and point-to-point. Furthermore since the MAC address is not included in the IEEE 802.16 frame headers, so typical usage of the Address Resolution Protocol (ARP) does not apply. Appropriate updates thus need to be done following [19] and [17] in order to make the module compliant to that. In particular, the IP and Ethernet specific subparts of the convergence sublayer has to be updated.

Currently the schedulers implement a simple FCFS based algorithm. The standard does not specify a definitive scheduling algorithm and leaves this up to the manufacturers to decide which algorithm to adopt. A lot of research is currently going on this topic and a number of algorithms have been proposed. A potential future work therefore is to update schedulers with a more sophisticated algorithm. Similarly the bandwidth management mechanism may also be updated with a more efficient algorithm.

Work on a propagation/error model at the PHY layer is currently under way. Another future work is to add support in the BS to dynamically update burst profile information, which is actually dependent on the implementation of the propagation/error model.

Current implementation is based on 802.16-2004 standard. In order to extend the module with the enhancements introduced by $802.16 \mathrm{e}$ (802.16e-2005) standard, some updates would be required at the PHY layer (802.16e revises OFDMA PHY to support mobility) as well as at the MAC layer. 


\section{CONCLUSIONS}

This paper presented detailed design and implementation of an IEEE 802.16 WiMAX module for the recently released ns-3 simulator. The proposed module implements the PMP mode and two different PHY layers: a basic PHY layer that simply forwards bursts received from the MAC layer and a more complete WirelessMAN-OFDM PHY that follows the standard. Module's design thoroughly follows the object-oriented software development methodology and utilizes Unified Modeling Language (UML) for modeling. High attention has been put to come up with a detailed and standard compliant implementation and to design an API that facilitates reusability, scalability and maintainability of software. The module implements key components of WiMAX MAC, including QoS scheduling services, link manager, service flow manager, simple priority/FCFS based schedulers, and bandwidth manager. Benefiting from the features of ns-3, the module enables detailed tracing, simulation of real TCP/IP-stack, emulation, and additions of new scenarios using the Helper API. We hope this module contributes to the scientific society and facilitates in evaluating and designing WiMAX systems. The paper also discussed a number of limitations of our module including absence of a full classifier to map incoming packets to appropriate connections. Future work focuses on implementation of convergence sublayer for IP over IEEE 802.16 applications and updating schedulers with more efficient algorithms.

\section{ACKNOWLEDGMENTS}

The authors would like to thank Fabio Cristiani of Università degli Studi di Napoli Federico II and Kavé Salamatian of LIP6 (Laboratoire d'informatique de Paris 6) for contributing with their OFDM PHY work. We would also like to thank Mathieu Lacage, our colleague at INRIA Sophia Antipolis and one of the lead developers of ns-3 project, for his valuable time and suggestions during the early stages of the development.

\section{REFERENCES}

[1] IEEE Std. 802.16-2004, IEEE Standard for Local and Metropolitan Area Networks - Part 16: Air Interface for Fixed Broadband Wireless Access Systems. October 2004.

[2] DOCSIS (Data Over Cable System Interface Specification) Research Project, http://www.cs.clemson.edu/ jmarty/docsis.html, July 2005

[3] IEEE Std. 802.16e-2005, IEEE Standard for Local and Metropolitan Area Networks - Part 16: Air Interface for Fixed Broadband Wireless Access Systems Amendment 2: Physical and Medium Access Control Layers for Combined Fixed and Mobile Operation in Licensed Bands. February 2006.

[4] The Network Simulator ns-2 NIST add-on - IEEE 802.16 model (MAC+PHY). Technical report, National Institute of Standards and Technology, June 2007.

[5] IT ++ - Library of mathematical, signal processing and communication routines, http://itpp.sourceforge.net/, July 2008.

[6] The network simulator - ns-2, http://www.isi.edu/nsnam/ns/, October 2008.
[7] The ns-3 network simulator, http://www.nsnam.org/, October 2008.

[8] WiMAX Forum System Level Simulator NS-2 MAC+PHY Add-On for WiMAX (IEEE 802.16). Technical report, WiMAX Forum, July 2008.

[9] A. Belghith and L. Nuaymi. Design and Implementation of a QoS-included WiMAX Module for ns-2 Simulator. In 2nd International Conference on Simulation Tools and Techniques (SIMUTools'09). ACM, March 2008.

[10] J. F. Borina and N. L. da Fonseca. WiMAX Module for the ns-2 Simulator. In 18th Annual IEEE International Symposium on Personal, Indoor and Mobile Radio Communications (PIMRC'Or). IEEE, September 2007.

[11] J. Chen, C.-C. Wang, F. C.-D. Tsai, C.-W. Chang, S.-S. Liu, J. Guo, W.-J. Lien, J.-H. Sum, and C.-H. Hung. Design and Implementation of WiMAX Module for ns-2 Simulator. In 1st International Conference on Performance Evaluation Methodologies and Tools (VALUETOOLS'06). ACM, October 2006.

[12] F. Cristiani. Simulation of WiMAX Physical Layer. Technical report, Università degli Studi di Napoli Federico II, Italy, December 2007.

[13] C. Eklund, R. B. Marks, and K. L. Standwood. IEEE Standard 802.16: A Technical Overview of the WirelessMAN Air Interface for Broadband Wireless Access. IEEE Communications Magazine, 40(6):98-107, June 2002.

[14] T. R. Henderson, M. Lacage, and G. F. Riley. Network Simulations with the ns-3 Simulator. Demo paper at ACM SIGCOMM'08, August 2008.

[15] C. Hoymann. Analysis and Performance Evaluation of the OFDM-based Metropolitan Area Network IEEE 802.16. Computer Networks, Selected Papers from the European Wireless 2004 Conference, 49(3):341-36, October 2005.

[16] J. Jee, S. Madanapalli, and J. Mandin. IP over IEEE 802.16 Problem Statement and Goals. RFC 5154, April 2008.

[17] H. Jeon, M. Riegel, and S. Jeong. Transmission of IP over Ethernet over IEEE 802.16 Networks draft-ietf-16ng-ip-over-ethernet-over-802.16-06.txt. IETF Draft, April 2008.

[18] C. Larman. Applying UML and Patterns: An Introduction to Object-Oriented Analysis and Design and Iterative Development. Prentice Hall, third edition, 2004.

[19] S. Madanapalli, S. D. Park, and S. Chakrabarti. Transmission of IPv4 packets over IEEE 802.16's IP Convergence Sublayer draft-ietf-16ng-ipv4-over-802-dot-16-ipcs-03.txt. IETF Draft, July 2008.

[20] L. Nuaymi. WiMAX: Technology for Broadband Wireless Access. John Wiley \& Sons, 2007. 